of these latter, one died of shock. In $188 \pm$ there were three Spanton's operations, with one recurrence. The instrument used throughout this and following years was a straight shaft with a spiral point, by which the pillars are alternately pierced. I described the operation in the Edinburgh Medical Journal, and published full details of the cases in the Indian Medical Gazette for 1883, so will not recapitulate my experience. In 1885 I performed four Spanton's operations, with one recurrence after a year; and two other surgeons also operated on two cases, which rapidly recurred. Last year (1886) eleven Spanton's operations were performed, of which one recurred. This year, up to date, eight screw operations have been performed, of which two were followed by reappearance of the hernia. In one the recurrence was sudden and complete, with some pain. On the same day 1 performed herniotomy, excised the sac, and sutured the pillars. Another case of excision has since been also successfully performed. The total may thus be tabulated: Screw operations, with Mr. Dunnet Spanton's or the author's modified instrument, forty-eight cases (seven of these are known to have recurred); operations with ligature of the neck, and usually excision of the sac, seven (of these two died).

On these cases I will offer but very few remarks. With regard to the only really radical procedure, that of removal of the sac with suture of the canal, it is a somewhat severe measure. Without selection of cases, 1 doubt whether the mortality would be less than 5 per cent. By careful selection of cases it might not exceed 1 or 2 per cent. With only the latter mortality this operation for the radical cure of reducible hernia is justifiable; but the operator ought to avoid exposing old or weakly persons to an operation which is usually followed by more or less suppuration in the scrotum, and which is liable to graver complications. consider Spanton's operation a less certain cure, but cannot speak positively on this point. During the last year I have usually removed the screw at the time, merely using it as an instrument for suturing the pillars and neck of the sac with silver wire, which has been left in. But this method seems to me less secure than that of leaving the instrument in for a week or until it loosens. This operation appears free from danger to life; in fact, the statistics collected by Mr. Spanton prove it to be so. Like Wood's operation, the success depends on the skilful application of the instrument. There is perhaps a "knack" in it, which many surgeons without considerable practice might fail to acquire. This should tell against the general adoption of the operation, but affords no ground for decrying its actual achievements. It is, moreover, a simple operation, and one in which the beginner is likely to fail from over-caution rather than otherwise, the operation being simply inert. In my first thirty cases it is likely that 20 per cent. recurred; in my next thirty this percentage is likely to be halved. Any who are interested in this subject should refer to Mr. Spanton's papers in the Medical Times, 1885, or to my contributions before referred to.

Kashmir, India.

\section{HYDATID DISEASE OF THE CONVEX} SURFACE OF THE LIVER,

\section{DISCHARGING INTO THE PLEURA AND AFFECTING} IT SECONDARILY.

BY H. HANDFORD, M.D., M.R.C.P., PHYSICLAN TO THE NOTTINGHAM GENERAI HOSPITAX.

THe history of the case is shortly as follows:-A man aged sixty-one, a lacemaker, was admitted under my care into the Nottingham General Hospital, in November, 1886, complaining of troublesome cough, slight muco-purulent sputum, and great shortness of breath, especially on exertion. $\mathrm{He}$ was well nourished, and had been a strong man, except for some winter cough for the last few years. His present illness had begun three months previously, and he had been sent into the hospital, with a diagnosis of chronic pleuritic effusion, for the purpose of being tapped. He had the signs of a moderate pleuritic effusion on the right side, but the vocal resonance and tremitus were very little diminished. The liver was not much pushed down, neither was it appreciably enlarged or irregular. $\mathrm{He}$ was aspirated, and thirty-four ounces of opaque semi-purulent thuid withdrawn. It was highly albuminous, but did not gelatinise on standing, showing the absence of any large amount of fibrine. Under the microscope very few pus cells were found and no hooklets or membranes, or anything suggestive of hydatid origin. The opacity appeared to be due not so much to pus as to the fatty degeneration of insoluble albumen - that is to say, of a highly fibrinous and albuminous: exudation. The chest was aspirated twice again, at intervals of about three weeks, and after two months he was discharged practically well. He worked at his trade for six or eight months, and began to get short of breath again and. to be troubled with cough in August, 1887. In the end of September I was asked to see him in consultation with $\mathrm{my}$ friend Dr. Stafford. The patient was extremely ill, with the right side almost motionless and dull up to the clavicle. He had the most urgent dyspnce, febrile temperature, and coated tongue. Next day he became an in-patient. again, and about fifty ounces of pus were removed by aspiration. This was examined microscopically, but presented no unusual appearance. There were no hooklets and no membranes. Rather more than a week later, when he had somewhat recovered strength, the chest, which had filled again, was opened by a free incision by my colleague, Mr. Wright. The opening was made in the seventh space, a little behind the posterior axillary line. The ribs were so close together that a small portion of bone had to be removed in order to admit a full-sized indiarubber drainage tube. After five or six days numerous hydatid vesicles appeared in the wound and on the dressings. Their true character was verified microscopically by the laminated hyaline appearance of the cyst walls, and by the presence of numerous hooklets. The cysts continued to be discharged almost daily for about a month; but for ten days or more before his death, which occurred from asthenia, the discharge of hydatids ceased. There never had been any noticeable enlargement of the liver. At the inspection a thick-walled, irregular, indurated, suppurating cavity, large enough to contain about six ounces, was found in the convex surface of the liver, chiefly in the right lobe, but partly in the left, immediately below the diaphragm. It communicated by a. smooth, rounded opening, that would just admit the indexfinger, with the right pleural cavity. In the pleura, at the root of the collapsed right lung, was a single hydatid cyst the size of a walnut. This was the only trace of hydatid left. There was no evidence of there having been any growth of hydatids in the lung, or any extensive affection of the pleura. The majority of the hydatids discharged through the chest wall-they numbered upwards of 100-must have come from the liver through the opening in the diaphragm mentioned above. The disease was cured, with the exception of the one solitary cyst at the root of the lung, but the patient had not strength to enable tissue repair to progress properly.

With regard to the diagnosis, I should say that the dependence of the chest affection on hydatid disease was not suspected till the vesicles were discharged, and then their origin from the liver was thought probable. The only difficulty during life in accepting a hepatic origin for the cysts was the total absence of bile staining. In a case of hydatid disease of th 3 liver in a lad who was under my care in 1879 , and which discharged through the lung, the numerous and large cyst walls which were coughed up were all stained of a bright orange-yellow colour. I have kept the patient under observation more or less ever since, and he is in robust health at the present time. With regard to the relation of the pleural effusion to the hydatid disease, I think it probable that the effusion in November, 1886, was due to contiguity, and that the free communication with the pleura was not established till later.

The late Dr. Harvex.-At a special meeting of the County and City of Cork Medical and Surgical Association, held recently, the following resolution was passed unanimously:- "That we, the members of the County and City of Cork Medical and Surgical Association, having heard with extreme regret of the death of Dr. Joshua Harvey, the founder of the Medical Club, and for many years a very active and esteemed member of the medical profession in Cork, beg to convey our deep sympathy to his family on the death of one who stood so deservedly high in the estimation both of the public and his professiona? brethren." 